\title{
Remaining Life-Time Assessment of Gear Box using Regression Model
}

\author{
A. Joshuva', V. Sugumaran ${ }^{1 *}$, M. Amarnath ${ }^{2}$ and Sang-Kwon Lee ${ }^{3}$ \\ 1 School of Mechanical and Building Sciences (SMBS), VIT University, Chennai Campus, Vandalur-Kelambakkam \\ Road, Chennai-600127, Tamil Nadu, India; joshuva1991@gmail.com, v_sugu@yahoo.com \\ 2Department of Mechanical Engineering, Indian Institute of Information Technology Design and Manufacturing, \\ Airport Road, IIITDM Jabalpur Campus, Khamaria, Jabalpur - 482005, Madhya Pradesh, India; amarnath@iiitdm.in \\ 32Department of Mechanical Engineering, Inha University, Republic of Korea; sangkwon@inha.ac.kr
}

\begin{abstract}
Objectives: The main objective of this study is to develop a model which can able to predict the remaining life time working of a gearbox using vibration signals. Method: This study is considered as a machine learning problem which consists of three phases, namely feature extraction, feature selection and feature classification. In this research, histogram features are extracted from vibration signals, feature selection are carried out using J48 algorithm and different regression models were built to predict the reaming lifetime assessment of a gearbox. Findings: In this study, the J48 algorithm was used and the regression was found to be 0.8944 for Gaussian model. This is a novel approach to finding the life prediction of gearbox using histogram and regression model. Improvements: This algorithm is applicable for real-time analysis and further the condition monitoring can be carried out using different algorithms with less computation time.
\end{abstract}

Keywords: Assessment, Fault Diagnosis, Gearbox, Histogram Features, Life Time, Multiple Regression, Sound Signals

\section{Introduction}

Gearbox is widely used component in the machinery for power transmission, which uses gears and gear trains to provide speed and torque conversion from a rotating power source to another device. Over the period of operating time, the gearbox may get damage, particularly to gear tooth. This reduces the lack of productivity. The life span of a gearbox can be predicted using condition monitoring technique. This lifetime prediction is used to replace the gearbox before it is worn out completely to prevent the machines from damage ${ }^{1}$. Many researchers have made a study of fault diagnosis of gears through sound and vibration signals and analyzed their signals through pattern recognition ${ }^{2-4}$.

There are two main predictive analysis types used to classify the faults and their signals in fault diagnosis process; they are classification and regression. A classifi- cation analysis is an ordered set of related categories used to group data according to its similarities ${ }^{5}$. It consists of codes and descriptions and allows survey responses to be put into meaningful categories in order to produce useful data. Regression analysis is a statistical technique which used to determine the strength of the relationship between one dependent variable and a series of other changing variables (known as independent variables) ${ }^{6}$. In this paper, regression had been chosen.

There are different types of regression models available to name a few, linear regression, simple linear regression, logistic regression, nonlinear regression, nonparametric regression, robust regression, stepwise regression, local regression etc. In this study multiple regressions models like Gaussian Processes (GP), Simple Linear Regression (SLR), Isotonic Regression (IR), Least Median of Squares (LMS), Multilayer Perceptron (MLP), Pace Regression (PR), Radial Basis Function (RBF) network, Linear

*Author for correspondence 
Regression (LR) and Sequential Minimal Optimization (SMO) regression were chosen for study.

The main purpose of multiple regressions is to analyze the relationship between metric or dichotomous independent variables and a metric dependent variable. Regression models were widely used in many studies to compare and suggest the better model which used to eliminate the failures in the machinery parts. A new feature for monitoring the condition of gearboxes is nonstationary operating conditions ${ }^{\mathrm{z}}$. The proper planetary gearbox condition is connected with perturbation of arm rotation ${ }^{8}$, where an arm rotation gives rise to a specific vibration signal whose properties are depicted by a Short-Time Fourier transform (STFT) and Wigner-Ville distribution

Prediction of work piece hardness using Artificial Neural Network (ANN). In this study, they predicted hardness compared with feed current ${ }^{9}$. They used LR analysis and obtained correlation coefficient of 0.958 . Yimin Shao et al. $\frac{10}{}$ did their study on fault prognosis and diagnosis of an automotive rear axle gear using a RBF-BP neural network using vibration signals. For an application they found the classification of different faults.

Detecting cracks in spur gears using regression trees with vibration signals ${ }^{11}$. They used LR, logistic regression, boosted regression tree and compared the better regression model for fault diagnosis. Vibration based condition monitoring of a multistage epicyclic gearbox in lifting cranes using autoregressive modeling and done accelerated lifetime test on the planetary gearbox $\stackrel{12}{2} \stackrel{13}{ }$ Multimodal deep support vector classification with homologous features and its application to gearbox fault diagnosis using vibration signals.

A gear fault diagnosis method based on local mean decomposition and generalized morphological fractal dimensions by using vibration signals and kernel fuzzy ${ }^{14}$. Many of the above works cited are based on vibration signals. In this paper, sound signals have been taken as a parameter for remaining lifetime assessment of the gearbox. The vibration signals can also be considered for this fault diagnosis; however the cost of the piezoelectric transducer is high compared to the microphone. Hence, sound signal was taken. Here, the histogram features were used.

The histogram feature have been successfully used as features in many fault diagnostic studies such as tool condition monitoring $\frac{15}{}$, centrifugal pump fault diagnosis $\frac{16}{6}$ , bearing fault diagnosis $\frac{17}{17}$ etc. The histogram features ${ }^{18}$ have different values for different hours of run time and this property can be used for regression model. Figure 1 shows the methodology flow chart of gear fault diagnosis.

The contribution of the study is as follows:

- In this paper, sound signals were taken from the good and faulty gearbox with the help of a microphone.

- The histogram features were taken and the feature selection was done by $\mathrm{J} 48$ decision tree algorithm.

- The multiple regressions were used to analyse the result. Various regression models were used and the better model was suggested for lifetime assessment of the gearbox.

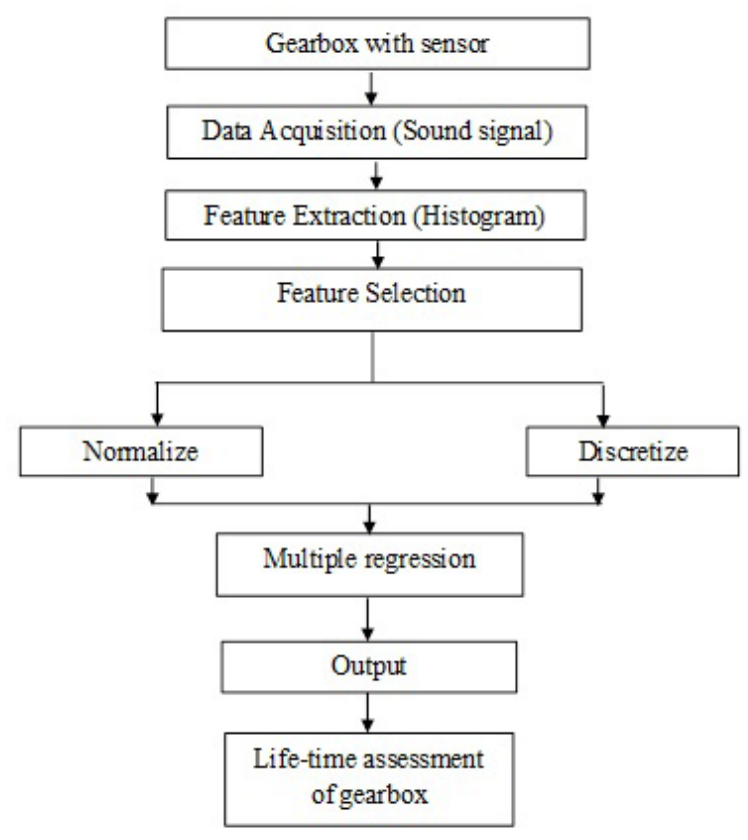

Figure 1. Methodology.

\section{Experimental Studies}

The experimental setup consists of $0.75 \mathrm{~kW} \mathrm{DC}$ motor and a single stage spur gearbox with a pair of gears mounted on two parallel shafts. The driving pinion had 24 teeth and driven gear had 25 teeth. The pinion was considered for fault assessment. Table 1 gives the detailed specifications of the gear box and test conditions. The DC motor rotation was controlled by a variable speed controller, which was used to drive the input shaft. The eddy current magnetic brake was used to provide torque load 
to the gearbox, this device has a maximum torque capacity of $12 \mathrm{Nm}$.

Table 1. Gear test rig specifications

\begin{tabular}{|c|c|c|}
\hline Parameter & Pinion & Gear \\
\hline Number of teeth & 24 & 25 \\
\hline Deport angle & 0.00 & 0.00 \\
\hline Pressure angle & $20^{\circ}$ & $20^{\circ}$ \\
\hline Height $(\mathrm{mm})$ & 6.53 & 6.53 \\
\hline Module & 3 & 3 \\
\hline Face width $(\mathrm{mm})$ & 30 & 30 \\
\hline $\begin{array}{l}\text { Pitch diameter } \\
(\mathrm{mm})\end{array}$ & 72.72 & 75.75 \\
\hline $\begin{array}{l}\text { Diameter of base } \\
(\mathrm{mm})\end{array}$ & 68.34 & 71.18 \\
\hline $\begin{array}{l}\text { Diameter of head } \\
(\mathrm{mm})\end{array}$ & 78.78 & 81.81 \\
\hline $\begin{array}{l}\text { Pinion Speed } \\
\text { (RPM) }\end{array}$ & 450 & \\
\hline $\begin{array}{l}\text { Torque on pinion } \\
\text { shaft }(\mathrm{Nm})\end{array}$ & $0-12$ & \\
\hline \multicolumn{3}{|c|}{ Material Properties of gears } \\
\hline Material & \multicolumn{2}{|c|}{ SM 45 C } \\
\hline $\begin{array}{l}\text { Brinell hardness } \\
\text { number Hardness } \\
\text { ( HRB) }\end{array}$ & \multicolumn{2}{|c|}{167} \\
\hline Poisson's ratio & \multicolumn{2}{|c|}{0.3} \\
\hline Young's modulus & \multicolumn{2}{|c|}{$2 \times 10^{5} \mathrm{~N} / \mathrm{mm}^{2}$} \\
\hline
\end{tabular}

The vibration and sound signals were acquired using a Bruel \& Kjaer 4189 microphone. In this study, sound signal has been used to predict the lifetime assessment of the gearbox. A commercial data acquisition system LMS SCADASIII was used to process sound and vibration signals. These signals were sampled at $8.2 \mathrm{kHz}$, acquired simultaneously and stored in a personal computer for post processing.

The gearbox is made to run for 900 hours for the lifetime prediction. The microphone was used to acquire sound signals from the gearbox which was installed in an acoustic rigid anechoic chamber. The microphone was kept at a distance of $5.5 \mathrm{~cm}-6 \mathrm{~cm}$ in the vicinity of the input shaft, this position was considered after many trials near to field condition. The experimental setup is shown in Figure $2 \frac{19}{}$.

Sound signals were taken from the good and different faulty conditions with the help of a microphone. The sound signals were obtained corresponding to good and faulty gearbox conditions which are used in determining the lifetime assessment. If the time domain sampled signals are used directly as inputs to the regression model, then the number of samples should be constant for given sampling rate. The number of samples which obtained is the function of rotation of speed of shaft; hence it can't be used directly as an input to the model. However, some features have to be extracted before the classification process $^{20}$.

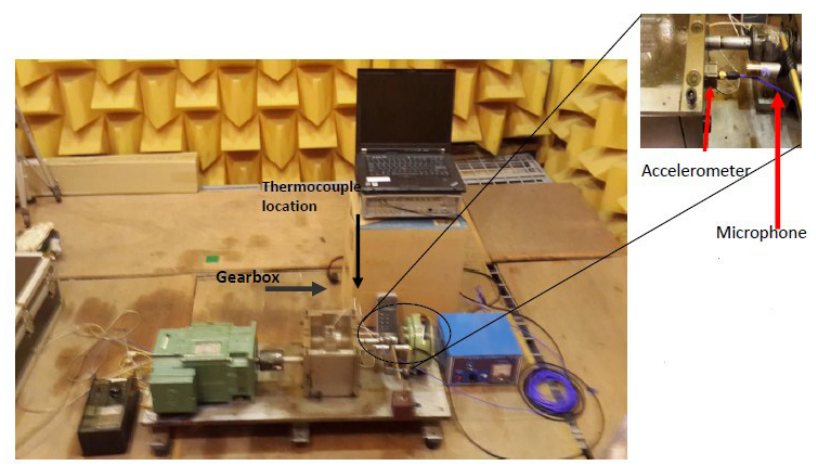

Figure 2. Experimental setup.

By investigating these set of signals, the histogram can be plotted with optimum bin size. J48 decision tree algorithm was used as a feature selection tool. Histogram features give the better plot of various ranges of amplitudes in sampled signals. It can be plotted for different hours of run for gearbox with the help of bins. The bin range should fit within the amplitude range of signals which are obtained from all conditions of bearings. With the maximum and the minimum values, the range was considered for plotting of the histogram. The maximum value and the minimum value of the range is ' 138.5262 ' and ' 114.313 ' respectively. This range was divided into 2 to 30 parts to form bins.

\section{Feature Selection}

The range of the bin is to be fixed so that the amplitude of the bin is different for various remaining lifetime of the gear box. The classification accuracy was computed for various numbers of bins and has been shown in the Table 2. In these various bin sets, the highest classification accuracy of $63.88 \%$ was achieved when the number of bins was 11 .

By using this bin 11 , the remaining study has been carried out. The feature selection process is then carried out 
with features extracted with 11 bins using J48 algorithm. It is a tree algorithm which consists of a root with various nodes and branches. The histogram features provide the input to the algorithm which intern produces the decision tree output as shown in Figure 3.

Table 2. Bin sets with the classification accuracy using histogram features

\begin{tabular}{|c|c|}
\hline No. of bins & Classification Accuracy (\%) \\
\hline 2 & 25.37 \\
\hline 3 & 29.25 \\
\hline 4 & 31.29 \\
\hline 5 & 30.55 \\
\hline 6 & 29.81 \\
\hline 7 & 33.51 \\
\hline 8 & 30.92 \\
\hline 9 & 52.59 \\
\hline 10 & 28.70 \\
\hline 11 & 63.88 \\
\hline 12 & 29.07 \\
\hline 13 & 46.66 \\
\hline 14 & 31.11 \\
\hline 15 & 45.92 \\
\hline 16 & 43.70 \\
\hline 17 & 37.77 \\
\hline 18 & 54.25 \\
\hline 19 & 36.85 \\
\hline 20 & 58.14 \\
\hline 21 & 32.59 \\
\hline 22 & 63.14 \\
\hline 23 & 39.44 \\
\hline 24 & 52.40 \\
\hline 25 & 49.81 \\
\hline 26 & 46.85 \\
\hline 27 & 52.77 \\
\hline 28 & 46.11 \\
\hline 29 & 55.18 \\
\hline 30 & 42.592 \\
\hline
\end{tabular}

The decision tree $e^{21}$ shows the features of the gearbox in descending order of information content. Hence the top node of the tree is the most important feature in the regression model. With the top node ' $X 7$ ' as the main feature, the other features with hours have been performed to check which combination provides high classification accuracy for the feature selection. The combination of $X 5, X 6, X 7, X 8, X 9$ and $X 10$ gave classification accuracy of $64.6296 \%$ when compared with other feature combinations as shown in the Table 3.

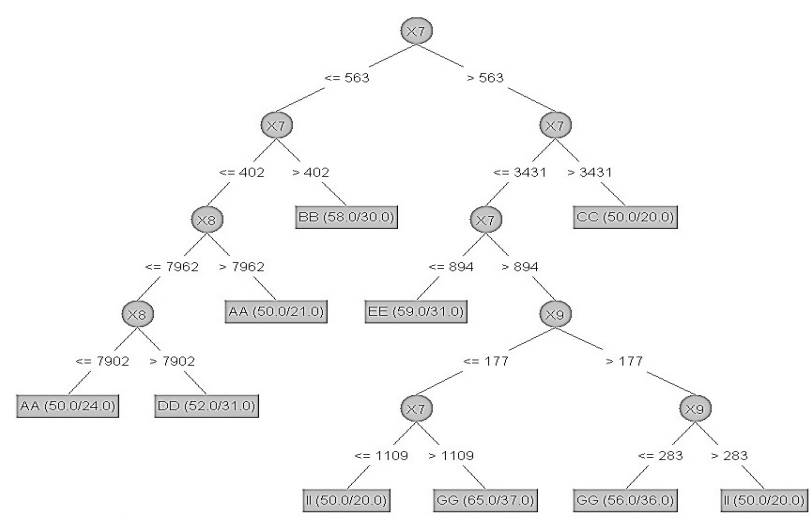

Figure 3. J48 tree algorithm.

Table 3. Combination of the features with different parameters used for feature classification

\begin{tabular}{|l|l|}
\hline Combination & $\begin{array}{l}\text { Correctly } \\
\text { Classified } \\
\text { Instances }(\%)\end{array}$ \\
\hline$X 7+$ Hours & 54.4444 \\
\hline$X 7+X 8+$ Hours & 63.7037 \\
\hline$X 6+X 7+X 8+$ Hours & 63.1481 \\
\hline$X 6+X 7+X 8+X 9+$ Hours & 64.0741 \\
\hline$X 5+X 6+X 7+X 8+X 9+$ Hours & 64.2593 \\
\hline$X 5+X 6+X 7+X 8+X 9+X 10+$ Hours & $\mathbf{6 4 . 6 2 9 6}$ \\
\hline$X 4+X 5+X 6+X 7+X 8+X 9+X 10+$ Hours & 64.4444 \\
\hline$X 4+X 5+X 6+X 7+X 8+X 9+X 10+X 11+$ Hours & 64.0741 \\
\hline $\begin{array}{l}X 3+X 4+X 5+X 6+X 7+X 8+X 9+X 10+X 11+ \\
\text { Hours }\end{array}$ & 64.0741 \\
\hline $\begin{array}{l}X 3+X 4+X 5+X 6+X 7+X 8+X 9+X 10+X 11+X \\
12+\text { Hours }\end{array}$ & 63.8889 \\
\hline $\begin{array}{l}X 2+X 3+X 4+X 5+X 6+X 7+X 8+X 9+X 10+X 11 \\
+X 12+\text { Hours }\end{array}$ & 63.8889 \\
\hline $\begin{array}{l}X 1+X 2+X 3+X 4+X 5+X 6+X 7+X 8+X 9+X 10 \\
+X 11+X 12+\text { Hours }\end{array}$ & 63.8889 \\
\hline $\begin{array}{l}X 1+X 2+X 3+X 4+X 5+X 6+X 7+X 8+X 9+X 10 \\
+X 11+X 12+X 13+\text { Hours }\end{array}$ & 63.8889 \\
\hline
\end{tabular}

\section{Regression Model}

In statistics, regression analysis is a statistical process for estimating the relationships among variables. Its focus 
is on the relationship between a dependent variable and one or more independent variables. The performance of regression analysis methods in practice depends on the form of the data generating process, and how it relates to the regression approach is used ${ }^{22}$. Multiple regressions is simultaneously considered the influence of multiple explanatory variables on a response variable $Y$

$$
E(Y)=\alpha+\beta_{1} X_{1}+\cdots . .+\beta_{k} X_{k}
$$

Where $\alpha=E(Y)$ when $X_{1}=X_{2}=\ldots=X_{k}=0 . \beta_{1}, \beta_{2 \ldots} B_{k}$ are called partial regression coefficients. The purpose of regression is to analyze the relationship between metric or dichotomous independent variables and a metric dependent variable. If there is a relationship, using the information in the independent variables will improve the accuracy in predicting values for the dependent variable. The different regression types are discussed below.

\subsection{Gaussian Processes (GP)}

It is a stochastic process whose realizations consist of random values associated with every point in a range of times or space such that each such random variable has a normal distribution ${ }^{23}$. They are simple to implement, flexible and fully probabilistic models

$$
E\left(\exp \left(i \sum_{l=1}^{k} t_{l} X_{t l}\right)\right)=\exp \left(-\frac{1}{2} \sum_{l, j} \sigma_{l j} t_{l} t_{j}+i \sum_{l} \mu_{l} t_{l}\right)
$$

The numbers $\sigma_{l j}$ and $\mu_{l}$ can be shown to be the covariance's and means of the variables in the process.

\subsection{Isotonic Regression (IR)}

It's a numerical, analysis which involves finding a weighted least-squares fit $x$ ? $R^{n}$ to a vector $a \in R^{n}$ with weights vector $w \in R^{n}$ subject to a set of non-contradictory constraints of kind $x_{i \geq} x_{j}$. Isotonic regression ${ }^{24}$ is also sometimes referred to as monotonic regression. Correctly speaking, isotonic is used when the direction of the trend is strictly increasing, while monotonic could imply a trend that is either strictly increasing or strictly decreasing. IR under the $L_{p}$ for $p>0$ is defined as

$$
\min \sum_{i=1}^{n}\left|x_{i}-a_{i}\right| p
$$

\subsection{Least Median Squares (LMS)}

It is the method estimates the parameters by solving the nonlinear minimization problem. Ordinary Least
Squares (OLS or LS) fit the line by finding the intercept and slopes that minimize the sum of squared residuals (SSR) are found. More formally, the optimization problem looks like

$$
{ }_{b_{\mathbf{0}} b_{\mathbf{1}}}^{\min } S S R=\sum_{i=\mathbf{1}}^{n}\left(Y \rrbracket_{i}-\mathbb{8}\left(b_{0}+b_{1} X_{i}\right)\right)^{\mathbf{2}}
$$

Analytical solutions for the intercept and slope choice variables are easily calculated. "Legendre called it the method of least squares, and it became a cornerstone of statistics. In spite of its mathematical beauty and computational simplicity, this estimator is now being criticized more and more for its dramatic lack of robustness ${ }^{25}$.

\subsection{Multilayer Perceptron (MLP)}

An MLP is a network of simple neurons called perceptron. The basic concept of a single perceptron was introduced by Rosenblatt in $1958^{26}$. The perceptron computes a single output from multiple real-valued inputs by forming a linear combination according to its input weights and then possibly putting the output through some nonlinear activation function. Mathematically, this can be written as

$$
y=\varphi\left(\sum_{i=1}^{n} \omega_{i} x_{i}+b\right)=\varphi\left(\omega^{T} x+b\right)
$$

Where $\omega$ denotes the vector of weights, $X$ is the vector of inputs, $b$ is the bias and $\varphi$ is the activation function.

\subsection{Pace Regression (PR)}

It is optimal when the number of coefficients tends to infinity. It consists of a group of estimators that are either overall optimal or optimal under certain conditions. The current work on the PR theory, and therefore also this implementation, do not handle missing values and nonbinary nominal attributes ${ }^{27}$.

$$
\theta_{i}^{E B}=\frac{\int \theta f\left(x_{i} \mid \theta\right) d G_{k}(\theta)}{\int f\left(\left.x_{i}\right|^{\theta}\right) d G_{k}(\theta)}
$$

\subsection{Radial Basis Function (RBF) Network}

It is a real-valued function whose value depends only on the distance from the origin, so that $\emptyset(X)=\emptyset(\|X\|)$ ; or alternatively on the distance from any other point $c$, called a centre, so that $\emptyset(X, C)=\emptyset(\|X-C\|)$. It can also be interpreted as a rather simple single-layer type of ANN called a RPF network ${ }^{28}$, with the RPFs taking on the role of the activation functions of the network. 


$$
y(X)=\sum_{i=1}^{N} \omega_{i} \emptyset\left(\left\|X-X_{i}\right\|\right)
$$

\subsection{Simple Linear Regression (SLR)}

It is the least squares estimator of a LR model with a single explanatory variable ${ }^{29}$. In other words, SLR fits a straight line through the set of $n$ points in such a way that makes the sum of squared residuals of the model as small as possible.

$$
y=\alpha+\beta x
$$

\subsection{Linear Regression (LR)}

It is an approach for modelling the relationship between a scalar dependent variable $y$ and one or more explanatory variables denoted $X$. In LR, data is modelled using linear predictor functions, and unknown model parameters are estimated from the data. Such models are called linear models ${ }^{30}$. Most commonly, LR refers to a model in which the conditional mean of $y$ given the value of $X$ is an affine function of $X$. Thus the model takes the form

$$
\begin{aligned}
& \mathbf{y}_{\mathrm{i}}=\boldsymbol{\beta}_{1} \mathbf{x}_{11}+\ldots+\beta_{\mathrm{p}} \mathbf{x}_{\mathrm{ip}}+\varepsilon_{\mathrm{i}}=\mathrm{X}_{\mathrm{i}}^{\mathrm{T}} \boldsymbol{\beta}+\varepsilon_{1}, \\
& i=1, \ldots . n
\end{aligned}
$$

Where $T$ denotes the transpose, so that $X_{i}^{T} \beta$ is the inner product between vectors $x_{i}$ and $\beta$.

\subsection{Sequential Minimal Optimization (SMO) Regression}

SMO regression implements the support vector machine for regression. The parameters can be learned using various algorithms. The algorithm is selected by setting the regression optimizer ${ }^{31}$. The most popular algorithm and this is the default regression optimizer.

$$
f(x, \alpha, \beta)=\sum_{i=1}^{l} y_{i} \alpha_{i} K\left(x_{i}, x\right)+b
$$

\section{Results and Discussion}

In the present study, lifetime prediction of gearbox is carried out with the help of multiple regressions. The histogram feature extraction and feature selection were carried out. The several bins were created with the help of constant maximum and minimum value range. Then, the number of bins for which the maximum classification accuracy was obtained was chosen as the number of bins for the rest of the study. From Table 2, it happens to be 11 bins. The J48 algorithm was used for the purpose of feature selection ${ }^{32}$. The feature selection is used to reduce the features and the parameters which will lead to a simple system and fast fault diagnosis of the gearbox. Then, the regression analysis was performed by multiple regression models and the results are discussed in the following paragraph.

From Table $3, \quad X 5+X 6+X 7+X 8+X 9+X 10+$ Hours from bin 11 provides the maximum correctly classified instances of $64.6296 \%$ and this combination is chosen for the remaining study for the lifetime prediction. Various regression models are applied to this particular combination and the results are produced below. From Table 4, different regression models have been built. The independent variables were normalized and discretized for better result $\frac{33}{}$. Normalizes implies every single numeric value in the certain dataset (aside from the class characteristic, if it is set).

Table 4. Regression results

\begin{tabular}{|l|l|l|}
\hline \multicolumn{2}{|c|}{$X 5+X 6+X 7+X 8+X 9+X 10+$ Hours_Regression Model } \\
\hline Regression Model & $\begin{array}{l}\text { Correlation } \\
\text { coefficient } \\
\text { (Normalized) }\end{array}$ & $\begin{array}{l}\text { Correlation } \\
\text { coefficient } \\
\text { (Discretized })\end{array}$ \\
\hline Gaussian Processes & $\mathbf{0 . 8 6 1 9}$ & $\mathbf{0 . 8 9 4 4}$ \\
\hline Isotonic regression & 0.8263 & 0.8365 \\
\hline $\begin{array}{l}\text { Least median } \\
\text { squares }\end{array}$ & 0.4258 & 0.8844 \\
\hline LR & 0.4883 & 0.8933 \\
\hline $\begin{array}{l}\text { Multilayer } \\
\text { perceptron }\end{array}$ & 0.3703 & 0.8163 \\
\hline Pace regression & 0.5082 & 0.6125 \\
\hline RBF network & 0.3514 & 0.4153 \\
\hline SLR & 0.4929 & 0.5874 \\
\hline SMO regression & 0.4251 & 0.8821 \\
\hline
\end{tabular}

The subsequent values are by default in $[0,1]$ for the information used to compute the normalization intermissions. Discretize is an instance filter that discretizes a range of numeric attributes in the dataset into nominal attributes. Discretization is by simple binding and skips the class attribute if it is set. From Figure 4, GP give the maximum correlation coefficient of 0.8619 in normalized 
condition and 0.8944 in discretized condition. Thus GP are used to predict the remaining lifetime of the gearbox

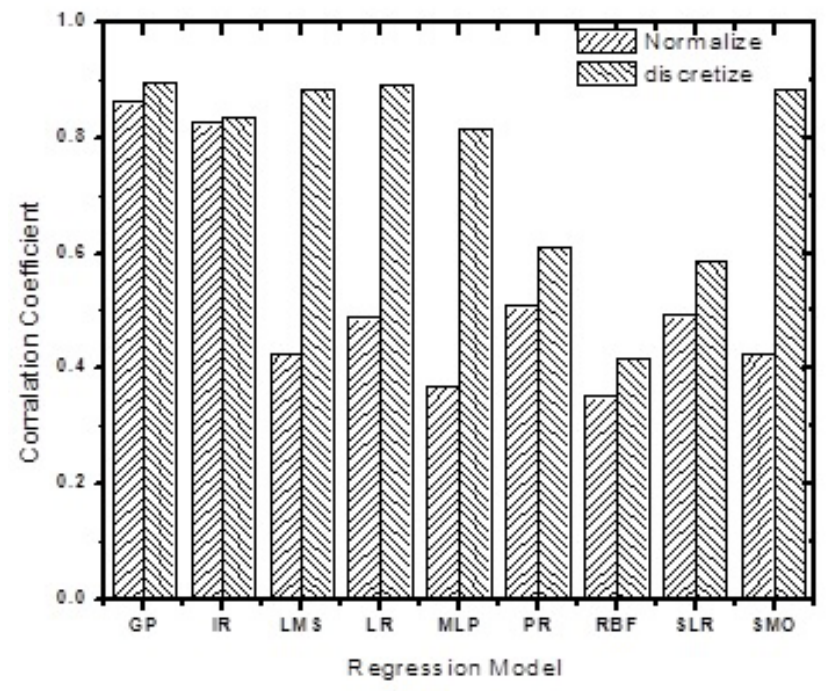

Figure 4. Correlation coefficient vs. regression model.

\section{Conclusion}

The main purpose of this study is to provide a best regression model for the life prediction of the gearbox. When comparing with several regression models, GP model has the highest correlation coefficient of 0.8944 . The mean absolute error found to be 0.0546 and root mean square error value is about 0.2019 . If the correlation coefficient value is closest to one, then it is the best suitable model for prediction problems. It has the very low mean absolute error and RMS error. From this, one can conclude that the GP model is capable of predicting the remaining life assessment of a gearbox when compared to other regression models.

\section{References}

1. Staszewski WJ, Worden K, Tomlinson GR. Time-frequency analysis in gearbox fault detection using the Wigner-Ville distribution and pattern recognition. Mechanical Systems and Signal Processing. 1997 Sep 30; 11(5):673-92.

2. Zheng H, Li Z, Chen X. Gear fault diagnosis based on continuous wavelet transform. Mechanical systems and signal processing. 2002 Mar 31; 16(2):447-57.

3. Widodo A, Yang BS. Support vector machine in machine condition monitoring and fault diagnosis. Mechanical Systems and Signal Processing. 2007 Aug 31; 21(6):2560-74.
4. Lei Y, Zuo MJ, He Z, Zi Y. A multidimensional hybrid intelligent method for gear fault diagnosis. Expert Systems with Applications. 2010 Mar 31; 37(2):1419-30.

5. Saravanan N, Ramachandran KI. Incipient gear box fault diagnosis using Discrete Wavelet Transform (DWT) for feature extraction and classification using Artificial Neural Network (ANN). Expert Systems with Applications. 2010 Jun 30; 37(6):4168-81.

6. Wang X, Makis V. Autoregressive model-based gear shaft fault diagnosis using the Kolmogorov-Smirnov test. Journal of Sound and Vibration. 2009 Nov 13; 327(3):413-23.

7. Bartelmus W, Zimroz R. A new feature for monitoring the condition of gearboxes in non-stationary operating conditions. Mechanical Systems and Signal Processing. 2009 Jul 31; 23(5):1528-34.

8. Bartelmus W, Zimroz R. Vibration condition monitoring of planetary gearbox under varying external load. Mechanical Systems and Signal Processing. 2009 Jan 31; 23(1):246-57.

9. Raj GBM, Sugumaran V. Prediction of work piece hardness using artificial neural network. International Journal of Design and Manufacturing Technology (IJDMT). 2010 Aug; 1(1):29-44.

10. Shao Y, Liang J, Gu F, Chen Z, Ball A. Fault prognosis and diagnosis of an automotive rear axle gear using a RBF-BP neural network. In Journal of Physics: Conference Series. IOP. 2011; 305(1):1-11.

11. Nanadic N, Ardis P, Hood A, Thurston M, Ghoshal A, Lewicki D. Comparative study of vibration condition indicators for detecting cracks in spur gears. In the 69th Annual Forum of American Helicopter Society (AHS) International, Phoenix, Arizona; 2013 May 21-3.

12. Assaad B, Eltabach M, Antoni J. Vibration based condition monitoring of a multistage epicyclic gearbox in lifting cranes. Mechanical Systems and Signal Processing. 2014 Jan 31; 42(1):351-67.

13. Li C, Sanchez RV, Zurita G, Cerrada M, Cabrera D, Vásquez RE. Multimodal deep support vector classification with homologous features and its application to gearbox fault diagnosis. Neurocomputing. 2015 Nov 30; 168:119-27.

14. Zheng Z, Jiang W, Wang Z, Zhu Y, Yang K. Gear fault diagnosis method based on local mean decomposition and generalized morphological fractal dimensions. Mechanism and Machine Theory. 2015 Sep 30; 91:151-67.

15. Elangovan M, Ramachandran KI, Sugumaran V. Studies on bayes classifier for condition monitoring of single point carbide tipped tool based on statistical and histogram features. Expert Systems with Applications. 2010 Mar 15; 37(3):2059-65.

16. Muralidharan V, Sugumaran V, Indira V. Fault diagnosis of monoblock centrifugal pump using SVM. Engineering 
Science and Technology, an International Journal. 2014 Sep 30; 17(3):152-7.

17. Lou X, Loparo KA. Bearing fault diagnosis based on wavelet transform and fuzzy inference. Mechanical systems and signal processing. 2004 Sep 30; 18(5):1077-95.

18. Lu W, Jiang W, Yuan G, Yan L. A gearbox fault diagnosis scheme based on near-field acoustic holography and spatial distribution features of sound field. Journal of Sound and Vibration. 2013 May 13; 332(10):2593-610.

19. Amarnath M, Krishna IRP. Empirical mode decomposition of acoustic signals for diagnosis of faults in gears and rolling element bearings. Science, Measurement and Technology, IET. 2012 Jul; 6(4):279-87.

20. Shao R, Hu W, Wang Y, Qi X. The fault feature extraction and classification of gear using principal component analysis and kernel principal component analysis based on the wavelet packet transform. Measurement. 2014 Aug 31; 54:118-32.

21. Sugumaran V, Jain D, Amarnath M, Kumar H. Fault diagnosis of helical gear box using decision tree through vibration signals. International Journal of Performability Engineering. 2013 Mar 1; 9(2):221-34.

22. Ali YH, Rahman RA, Hamzah RI. Regression modelling for spur gear condition monitoring through oil film thickness based on acoustic emission signal. Modern Applied Science. 2015 Aug 1; 9(8):21-8.

23. Zamanian AH, Ohadi A. Gear fault diagnosis based on Gaussian correlation of vibrations signals and wavelet coefficients. Applied Soft Computing. 2011 Dec 31; 11(8):4807-19.

24. Gorinevsky D, Kim SJ, Beard S, Boyd S, Gordon G. Optimal estimation of deterioration from diagnostic image sequence. Institute of Electrical and Electronics Engineers (IEEE) Transactions on Signal Processing. 2009 Mar; 57(3):1030-43.
25. Rousseeuw PJ. Least median of squares regression. Journal of the American statistical association. 1984 Dec 1; 79(388):871-80.

26. Rosenblatt F. The perceptron: a probabilistic model for information storage and organization in the brain. Psychological review. 1958 Nov; 65(6):386-408.

27. Çakır A, Çalış H, Küçüksille EU. Data mining approach for supply unbalance detection in induction motor. Expert Systems with Applications. 2009 Nov 30; 36(9):11808-13.

28. Gunn SR. Support vector machines for classification and regression. ISIS technical report; 1998 May 10. p. 1-53.

29. Bartelmus W, Zimroz R. A new feature for monitoring the condition of gearboxes in non-stationary operating conditions. Mechanical Systems and Signal Processing. 2009 Jul 31; 23(5):1528-34.

30. Feng Z, Zuo MJ, Chu F. Application of regularization dimension to gear damage assessment. Mechanical Systems and Signal Processing. 2010 May 31; 24(4):1081-98.

31. Shevade SK, Keerthi SS, Bhattacharyya C, Murthy KR. Improvements to the SMO algorithm for SVM regression. Institute of Electrical and Electronics Engineers (IEEE) Transactions on Neural Networks. 2000 Sep; 11(5):118893.

32. Joshuva A, Sugumaran V, Amarnath M. Selecting kernel function of support vector machine for fault diagnosis of roller bearings using sound signals through histogram features. International Journal of Applied Engineering Research. 2015; 10(68):482-7.

33. Ferrari T, Gini G. A new predictive model of Mutagenicity, with statistical analysis and validation using data-mining tools in WEKA. In Poster presented at SCARLET workshop; 2008 Apr 2. p. 2-4. 